\title{
Tailored drug therapy for mitigating drug-induced liver injury: is this the era of genetic screening?
}

"It is not too optimistic to expect that clinically handy and valuable genetic biomarkers that are predictive of the most important DILI will be available within the next decade."

Idiosyncratic drug-induced liver injury (DILI) is the single most frequent adverse drug reaction (ADR) causing drug withdrawal from the market. It accounts for more than a half of acute liver failure in the USA. The advent of genomics has made great progress in pharmacogenetics and pharmacogenomics. Many studies concerning genetic tests in DILI have emerged, raising the question of: is it beneficial to tailor drug treatment for alleviating DILI based on these genetic studies?

A compendium of positive genetic-association studies in DILI exist (Tabie 1). Most are related to the genetic polymorphisms of the drug metabolizing enzymes and some emphasize an association of HLA with DILI, but there are very little data regarding genetic polymorphisms of acquired immunity. Although a biomarker to predict idiosyncratic DILI is urgently needed, there is still no official recommendation for genotyping prior to initiating drug therapy in order to reduce the incidence of DILI.

Why is the application of pharmacogenomics in DILI and other ADRs unable to keep abreast of the recent progress in genomic knowledge and techniques? Many obstacles seem to convert the initial optimism and expectation into skepticism. While the genetic approach for predicting therapeutic efficacy and safety is a crucial way of establishing optimal personalized medicine, many complex problems emerge when interpreting available genetic data.

First, prospective clinical trails with genetic tests are an appropriate way to obtain beneficial information with little bias, but their value may be counteracted by their study designs. Through regular monitoring, they provide the opportunity to identify susceptible patients with a mild form of hepatotoxicity. Most trials warrant discontinuation of the drug when serum aminotransferase levels rise to three-five-times the upper limit of normal (ULN), or when serum bilirubin levels are elevated by two-threetimes the ULN. It is questionable whether these cohorts would have developed severe hepatotoxicity had therapy been continued. Therefore, cases with severe DILI or 'Hy's rule' are rarely detected, even in large clinical trials, because of the low incidence of severe cases and the early discontinuation of the involved drug.

Mild liver dysfunction cannot be neglected but is always less clinically significant and is easily confused with other background noise, such as fatty liver. For the US Drug-Induced Liver Injury Network (DILIN), the criterion for enrolling DILI patients is elevated serum transferase to more than five-times the ULN. The recruitment criteria and definition of DILI in genetic studies are diverse and include patients with mild DILI (Table 1).

Second, many DILI patients may have the adaptation phenomenon, which means that they resume normal or near-normal liver function without discontinuing the incriminating drug. Such adaptation is observed in more than a half of patients with anti-tuberculosis DILI. However, prospective trials cannot always discriminate adaptors owing to early drug withdrawal.

Furthermore, there is no consensus regarding the diagnosis of DILI in these genetic studies, such that the DILIN and the US FDA have recently endeavored to standardize the nomenclature and causality assessment through a series of workshops.

The other skepticism of genetic tests involves environmental factors that may alter the gene expression of drug-metabolizing enzymes and drug responses. These include age, gender, nutritional status, drug co-administration, alcohol consumption, food, chronic viral hepatitis infection, fatty liver and comorbidities. The triangle interaction of drug, host genetic factors and environmental factors forms an intricate network pertinent to human drug responses. However, decisions regarding which environmental factors are important and which may influence incriminated drugs remain inconsistent.

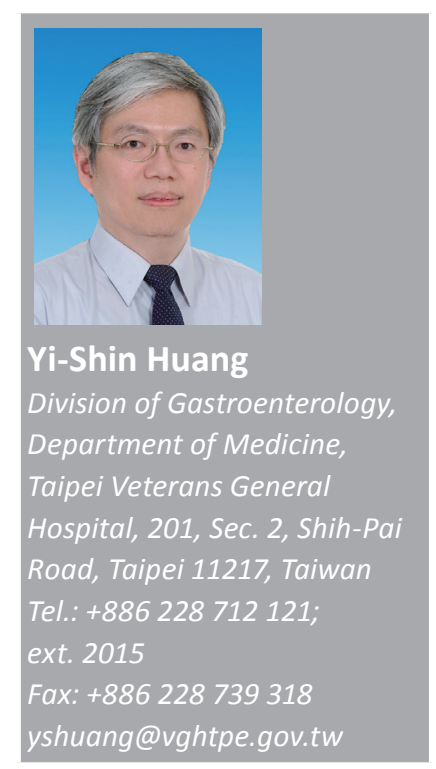

future medicine $^{\text {pis }} \mathbf{f s}$ 


\begin{tabular}{|c|c|c|}
\hline Drugs & High-risk genotypes & Ref. \\
\hline \multicolumn{3}{|c|}{ Drug metabolizing enzymes } \\
\hline Perhexiline & $C Y P 2 D 6 * 3, * 4, * 5$ and $* 6$ & [13] \\
\hline Carbamazepine & GSTM1 null & [14] \\
\hline Tacrine & GSTM1 and T1 null & {$[15]$} \\
\hline Troglitazone & GSTM1 and T1 null & [16] \\
\hline Diverse & GSTM1 and T1 null & [17] \\
\hline Methotrexate & GSTM1 non-null, RFC1 G80A & [18] \\
\hline Anti-TB drugs & NAT2*5, *6, *7, CYP2E1 c1/c1, MnSOD T/C or C/C, GSTM1 null & {$[2-11]$} \\
\hline Diclofenac & UGT2B7*2, CYP2C8 haplotype, ABCC2 C24T & [19] \\
\hline Tolcapone & UGT1A haplotype & [20] \\
\hline Indinavir & $\begin{array}{l}\text { UGT1A1*28, UGT1A3 T66C-UGT1A7 T57G } \\
\text { UGT1A1*6 }\end{array}$ & {$[21,22]$} \\
\hline Atazanavir & UGT1A1*28, UGT1A3 T66C-UGT1A7 T57G & [23] \\
\hline Azathioprine & $T P M T * 3 A$ and $* 3 C$ & [24] \\
\hline \multicolumn{3}{|c|}{ Immunological reactions } \\
\hline Flucloxacillin & $H L A-B * 5701$ & [1] \\
\hline $\begin{array}{l}\text { Amoxicillin- } \\
\text { clavulanate }\end{array}$ & $\begin{array}{l}H L A-D R B 1 * 1501-D R B 5 * 0101-D Q B 1 * 0602 \\
H L A-D Q A 1 * 0102-D Q B 1 * 0602\end{array}$ & {$[25,26]$} \\
\hline Ticlopidine & $H L A-A * 3303, H L A-D Q B 1 * 06$ & [27] \\
\hline Ximelagastran & $H L A-D R B 1 * 07, H L A-D Q A 1 * 02$ & [28] \\
\hline Anti-TB drugs & $H L A-D Q B 1 * 0201$ & [29] \\
\hline Diverse & $H L A D R B 1 * 15, D Q B 1 * 06$ & [30] \\
\hline Diclofenac & IL-10-627A and IL-4-590T & [31] \\
\hline Tacrine & IL-6-597A-572G-174G haplotype & [32] \\
\hline
\end{tabular}

In addition, although many association studies have already been conducted (TABLE 1), few present sufficient cases and controls to prove the power, and even fewer have been verified. The lack of reproducibility may be owing to diverse ethnicity, inclusion and exclusion criteria, and nongenetic factors, such as those previously mentioned.

Most of the studies in Table 1 are related to phase 1 (oxidation, reduction and toxification reactions) and phase 2 (conjugation and detoxification reactions) drug-metabolizing enzymes. The whole scope may be more comprehensive if genetic polymorphisms of phase 3 enzymes (transport and excretion) are included in future studies. Marked progress in high-throughput genotyping techniques has been made in the past few years that has decreased costs and increased the efficacy of genetic testing. It is now more convenient to analyze different genetic polymorphisms than it was before.

The major drawback for the clinical application of pharmacogenomics in DILI is its lack of robust association in larger sample sizes and well-defined phenotypes. Since the incidence of DILI may be as low as one in 1000 , or even in hundreds of thousands, international collaborations to recruit enough patients and controls with the same inclusion criteria for genetic studies may overcome this problem. However, the process of enrolling large patient and control numbers in the following genomic studies is costly and time consuming, such that finding a significant genetic signature of DILI is like searching for a needle in a haystack. In the beginning, it is better to focus on drugs that cause the most severe or most common DILI, such as anti-tuberculosis drugs, diclofenac and amoxicillin-clavulantate, in order for the trials to be more cost effective.

Most of the genetic studies in Table 1 are related to one or two functional genetic polymorphisms. However, since drug response is very complex and involves both known and unknown metabolic pathways, whole-genome studies are increasingly being considered to determine all variants associated with DILI and other ADRs.

Whole-genome association studies are now available using SNP chips such as those by Illumina $^{\circledR}$ (CA, USA) or Affymetrix ${ }^{\circledR}$ (CA, USA). Although the technology in whole-genome 
studies has tremendously improved in recent years, error rates are still problematic. A cluster of possibly associated genes shown on the SNP chips may look fancy but these may only dazzle and may not impress with solid information. Further identification and verification of the possible genes identified on SNP chips is mandatory and becomes useful after selecting limited significant genetic polymorphisms.

In contrast to the genome-wide study, the haplotype approach, like the international HapMap project initiated by the US NIH, may be a more feasible approach. Here, a few SNPs in each gene or chromosome in the haplotype are studied together.

Genotyping is not the only approach for assessing the risk of DILI. Metabonomic analysis of urine and serum from DILI patients, together with the proteomic study of serum, may identify a unique signature of hepatotoxicity. Gene expression is complicated and may be modified, augmented or suppressed by many factors, one of which is methylation (epigenetics). The transcriptomic method can be used to explore gene transcription and expression, which may explain the diverse expression of the same genetic trait.

\section{"...the potential for gaining insight into the genetic factors of DILI, and hence, for predicting and preventing it, is ... enormous."}

Relatively few pharmacogenetic or pharmacogenomic studies on DILI have so far been published compared with the large numbers of disease-risk pharmacogenomic studies. However, from published studies, the potential for gaining insight into the genetic factors of DILI, and hence, for predicting and preventing it, is still enormous. Recent findings on the association of flucloxacillin-induced hepatotoxicity and $H L A-B^{*} 5701$ are encouraging [1]. In this genome-wide association study using 866,399 markers, patients with $H L A-B^{*} 5701$ had a significantly higher risk of developing flucloxacillin-induced liver injury (odds ratio: 80.6). This highlights the value of genetic studies in understanding the mechanism of DILI and posits a successful model for further clinical application.

Anti-tuberculosis drug-induced hepatitis is the most prevalent DILI in Taiwan, India and many other countries [2-11]. One of the pharmacogenetic approaches for predicting DILI is genotyping $N$-acetyltransferase 2 (NAT2). It was reported that NAT2 slow acetylators may have a higher risk of anti-tuberculosis DILI than rapid acetylators in an Asian population [2,5,9,11]. Although this association is not verified in Caucasians, a prospective Isoniazid Dose Adjustment According to NAT2 Genotype (IDANAT2) trial has been launched to compare the safety and efficacy of isoniazid administration in Europe since 2008 [12]. In the test group, the dose of isoniazid has been adapted to $2.5 \mathrm{mg} / \mathrm{kg}, 5.0 \mathrm{mg} / \mathrm{kg}$ and $7.5 \mathrm{mg} / \mathrm{kg}$ for slow, intermediate and rapid acetylators, respectively. The standard isoniazid dose $(5.0 \mathrm{mg} / \mathrm{kg})$ is administered in the control group. Primary outcome measures are incidences of hepatotoxicity and early treatment failure. Regardless of the trial results, this kind of study is inspiring as a model that translates genetic testing to clinical practice.

"...together with accessible information on the human genome and other 'omics', there is the extraordinary opportunity to improve the clinical application of DILI via genetic testing."

Finally, to answer the query posed at the beginning of this editorial: is this the dawn of the era of genetic screening to mitigate DILI? Sadly, the answer for now is no. Nonetheless, together with accessible information on the human genome and other 'omics', there is an extraordinary opportunity to improve the clinical application of DILI via genetic testing. To pursue the high standard pharmacogenetics or pharmacogenomics studies, there is a need to recruit an international, multicenter, large sample size of patients and controls, with well-defined criteria of diagnosis and severity that consider both genetic and environmental factors. Although genetic data regarding a biomarker to predict DILI susceptibility and severity is still limited, this approach remains very promising since national and international collaborations are well on their way. It is not too optimistic to expect that clinically handy and valuable genetic biomarkers that are predictive of the most important DILI will be available within the next decade.

Financial \& competing interests disclosure

The author has no relevant affiliations or financial involvement with any organization or entity with a financial interest in or financial conflict with the subject matter or materials discussed in the manuscript. This includes employment, consultancies, honoraria, stock ownership or options, expert testimony, grants or patents received or pending, or royalties.

No writing assistance was utilized in the production of this manuscript. 


\section{Bibliography}

Papers of special note have been highlighted as:

- of interest

" of considerable interest

1 Daly AK, Donaldson PT, Bhatnagar P et al:: $H L A-B^{*} 5701$ genotype is a major determinant of drug-induced liver injury due to flucloxacillin. Nat. Genet. 41(7), 816-819 (2009).

"I Successful model of a genome-wide study to detect the biomarkers of drug-induced liver injury.

2 Huang YS, Chern HD, Su WJ et al.: Polymorphism of the $\mathrm{N}$-acetyltransferase 2 gene as a susceptibility risk factor of antituberculosis drug-induced hepatitis. Hepatology 35 (4), 883-889 (2002).

- Frequently cited paper to demonstrate slow acetylators as a risk factor of anti-tuberculosis hepatitis, using the NAT2 genotyping.

3 Huang. YS, Chern HD, Su WJ et al: Cytochrome P450 2E1 genotype and the susceptibility to antituberculosis drug-induced hepatitis. Hepatology 37(4), 924-930 (2003).

4 Huang YS, Su WJ, Huang YH et al:: Genetic polymorphisms of manganese superoxide dismutase, $\mathrm{NAD}(\mathrm{P}) \mathrm{H}$ : quinone oxidoreductase, glutathione $S$-transferase M1 and $\mathrm{T} 1$ and the susceptibility to drug-induced liver injury. J. Hepatol. 47(1), 128-134 (2007).

5 Ohno M, Yamaguchi I, Yamamoto I et al.: Slow $N$-acetyltransferase 2 genotype affects the incidence of isoniazid and rifampicin-induced hepatotoxicity. Int. J. Tuberc. Lung Dis. 4(3), 257-261 (2000).

6 Roy B, Chowdhury A, Kundu S et al.: Increased risk of antituberculosis druginduced hepatotoxicity in individuals with glutathione $S$-transferase M1 'null' mutation. J. Gastroenterol. Hepatol. 16(9), 1033-1037 (2001).

7 Roy B, Ghosh SK, Sutradhar D et al:: Predisposition of antituberculosis drug induced hepatotoxicity by cytochrome P450 2E1 genotype and haplotype in pediatric patients. J. Gastroenterol. Hepatol. 21(4), 784-786 (2006).

8 Vuilleumier N, Rossier MF, Chiappe A et al.: CYP2E1 genotype and isoniazid-induced hepatotoxicity in patients treated for latent tuberculosis. Eur. J. Clin. Pharmacol. 62(6), 423-429 (2006).

9 Cho HJ, Koh WJ, Ryu YJ et al.: Genetic polymorphisms of NAT2 and CYP2E1 associated with antituberculosis drug-induced hepatotoxicity in Korean patients with pulmonary tuberculosis. Tuberculosis $87(6)$, 551-556 (2007).
10 Leiro V, Fernandez-Villar A, Valverde D et al:: Influence of glutathione $S$-transferase $\mathrm{M} 1$ and T1 homozygous null mutations on the risk of antituberculosis drug-induced hepatotoxicity in a Caucasian population. Liver Int. 28(6), 835-839 (2008).

11 Kim SH, Kim SH, Bahn JW et al.: Genetic polymorphisms of drug-metabolizing enzymes and anti-TB drug-induced hepatitis. Pharmacogenomics 10(11), 1767-1779 (2009).

12 Tomalik-Scharte D, Lazar A, Fuhr U, Kirchheiner J: The clinical role of genetic polymorphisms in drug-metabolizing enzymes. Pharmacogenomics J. 8(1), 4-15 (2008).

13 Barclay ML, Sawyers SM, Begg EJ et al.: Correlation of CYP2D6 genotype with perhexiline phenotypic metabolizer status. Pharmacogenetics 13 (10), 627-632 (2003).

14 Ueda. K, Ishitsu T, Seo T et al:: Glutathione $S$-transferase M1 null genotype as a risk factor for carbamazepine-induced mild hepatotoxicity. Pharmacogenomics 8(5), 435-442 (2007).

15 Simon. T, Becquemont L, Mary-Krause M et al:: Combined glutathione-S-transferase M1 and T1 genetic polymorphism and tacrine hepatotoxicity. Clin. Pharmacol. Ther. 67(4), 432-437 (2000).

16 Watanabe I, Tomita A, Shimizu M et al.: A study to survey susceptible genetic factors responsible for troglitazone-associated hepatotoxicity in Japanese patients with Type 2 diabetes mellitus. Clin. Pharmacol. Ther. 73(5), 435-455 (2003).

17 Lucena MI, Andrade RJ, Martínez C et al: Glutathione-S-transferase M1 and T1 null genotypes as susceptibility factor for idiosyncratic drug-induced liver injury. Hepatology 48(2), 588-596 (2008).

18 Imanishi H, Okamura N, Yagi M et al.: Genetic polymorphisms associated with adverse events and elimination of methotrexate in childhood acute lymphoblastic leukemia and malignant lymphoma. J. Hum. Genet. 52(2), 166-171 (2007).

19 Daly AK, Aithal GP, Leathart JB, Swainsbury RA, Dang TS, Day CP: Genetic susceptibility to diclofenac-induced hepatotoxicity: contribution of $U G T 2 B 7$, $C Y P 2 C 8$, and $A B C C 2$ genotypes. Gastroenterology 132(1), 272-281 (2007).

20 Acuna. G, Foernzler D, Leong D et al:: Pharmacogenetic analysis of adverse drug effect reveals genetic variant for susceptibility to liver toxicity. Pharmacogenomics J. 2(5), 327-334 (2002).

21 Boyd MA, Srasuebkul P, Ruxrungtham K et al:: Relationship between hyperbilirubinaemia and
UDP-glucuronosyltransferase 1A1 (UGT1A1) polymorphism in adult HIV-infected Thai patients treated with indinavir. Pharmacogenet. Genomics 16(5), 321-329 (2006).

22 Lankisch TO, Behrens G, Ehmer U et al: Gilbert's syndrome and hyperbilirubinemia in protease inhibitor therapy: an extended haplotype of genetic variants increases risk in indinavir treatment. J. Hepatol. 50 (5), 1010-1018 (2009).

23 Lankisch TO, Moebius U, Wehmeier M et al:: Gilbert's disease and atazanavir: from phenotype to UDP-glucuronosyltransferase haplotype. Hepatology 44(5), 1324-1332 (2006).

24 Heckmann JM, Lambson EM, Little F, Owen EP: Thiopurine methyltransferase (TPMT) heterozygosity and enzyme activity as predictive tests for the development of azathioprine-related adverse events. J. Neurol. Sci. 231(1-2), 71-80 (2005).

25 Hautekeete ML, Horsmans Y, Van Waeyenberge $\mathrm{C}$ et al.: HLA association of amoxicillin-clavulanate-induced hepatitis. Gastroenterology 117(5), 1181-1186 (1999).

26 O’Donohue. J, Oien KA, Donaldson P et al.: Co-amoxiclav jaundice: clinical and histological features and HLA class II association. Gut 47, 717-720 (2000).

27 Hirata K, Takagi H, Yamamoto M et al.: Ticlopidine-induced hepatotoxicity is associated with specific human leukocyte antigen genomic subtypes in Japanese patients: a preliminary case-control study. Pharmacogenomics J. 8(1), 29-33 (2008).

28 Kindmark. A, Jawaid A, Harbron CG et al.: Genome-wide pharmacogenetic investigation of a hepatic adverse event without clinical signs of immunopathology suggests an underlying immune pathogenesis. Pharmacogenomics J. 8(3), 186-195 (2008).

29 Sharma SK, Balamurugan A, Saha PK, Pandey RM, Mehra NK: Evaluation of clinical and immunogenetic risk factors for the development of hepatotoxicity during antituberculosis treatment. Am. J. Respir. Crit. Care Med. 166(7), 916-919 (2002).

30 Andrade. RJ, Lucena MI, Alonso A et al: HLA class II genotype influences the type of liver injury in drug-induced idiosyncratic liver disease. Hepatology 39(6), 1603-1612 (2004).

31 Aithal GP, Ramsay L, Daly AK et al.: Hepatic adducts, circulating antibodies, and cytokine polymorphisms in patients with diclofenac hepatotoxicity. Hepatology 39(5), 1430-1440 (2004).

32 Carr DF, Alfirevic A, Tugwood JD et al: Molecular and genetic association of interleukin- 6 in tacrine-induced hepatotoxicity. Pharmacogenet. Genomics 17(11), 961-972 (2007). 